\title{
Aposteldekret und antikes Vereinswesen
}

\author{
Gemeinschaft und ihre Ordnung \\ Hrsg. v. Markus Öhler
}

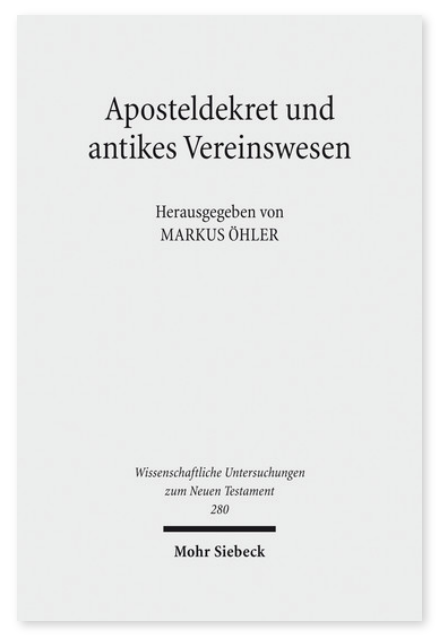

2011. VIII, 411 Seiten. WUNT I 280

ISBN 978-3-16-151779-2

DOI 10.1628/978-3-16-151779-2

eBook PDF $144,00 €$

ISBN 978-3-16-150363-4

Leinen $144,00 €$
Mit dem Aposteldekret präsentiert der Verfasser der Apostelgeschichte am Höhepunkt seiner Darstellung Bestimmungen zur Ordnung in der Gemeinde, deren historische und theologische Einordnung Rätsel aufgibt. Zu ihrer Lösung soll im vorliegenden Band ein Zusammenwirken über die Disziplinengrenzen beitragen. Die frühchristlichen Gemeinden, für die das Aposteldekret gedacht war, gehörten wie jüdische Synagogen zu dem weiten Bereich antiker Vereinigungen. Daher führt eine Verknüpfung der beiden Themenbereiche Aposteldekret und Vereinswesen, wie sie hier vorgenommen wird, zu neuen Einblicken und Erkenntnissen. Neben primär exegetischen Beiträgen und Untersuchungen zur Wirkungsgeschichte des Dekrets finden sich althistorische Einblicke in das antike Vereinswesen sowie Versuche, das Aposteldekret auf diesem Hintergrund deutlicher zu profilieren.

\section{Inhaltsübersicht}

Wilhelm Pratscher: Der Beitrag des Herrenbruders Jakobus zur Entstehung des Aposteldekrets - Friedrich Avemarie: Die jüdischen Wurzeln des Aposteldekrets. Lösbare und ungelöste Probleme - Hermut Löhr: »Unzucht«. Überlegungen zu einer Bestimmung der Jakobus-Klauseln im Aposteldekret sowie zu den Geltungsgründen von Normen frühchristlicher Ethik Richard S. Ascough: The Apostolic Decree of Acts and Greco-Roman Associations: Eating in the Shadow of the Roman Empire Loren T. Stuckenbruck: The »Cleansing « of the Gentiles: Background for the Rationale behind the Apostles' Decree in Acts 15 Matthias Klinghardt: Das Aposteldekret als kanonischer Integrationstext: Konstruktion und Begründung von Gemeinsinn Matti Myllykoski: Ohne Dekret: Das Götzenopferfleisch und die Frühgeschichte der Didache - Markus Lang: Die Bestimmungen des Aposteldekretes im zweiten und frühen dritten Jahrhundert - Andreas Bendlin: Associations, Sociality, and Roman Law: A New Interpretation of the cultores Dianae et Antinoi in Lanuvium - Andreas Gutsfeld: Vereinigungen bei Tisch. Zur Funktion von Banketten in paganen Vereinigungen der frühen Kaiserzeit - Julietta Steinhauer: Die Kultgemeinschaften der ägyptischen Gottheiten in Griechenland - Eva Ebel: Regeln von der Gemeinschaft für die Gemeinschaft? Das Aposteldekret und antike Vereinsregeln im Vergleich - Markus Öhler: Landsmannschaftliche Vereinigungen, christliche Gemeinden und das Aposteldekret

Markus Öhler Geboren 1967; Studium der Ev. Theologie und Universitätsassistent in Wien; FWF-Stipendiat in Tübingen; Gastprofessor in Hamburg; seit 2011 Professor am Institut für Neutestamentliche Wissenschaft der EvangelischTheologischen Fakultät der Universität Wien.

https://orcid.org/0000-0003-4294-9778

Jetzt bestellen:

https://mohrsiebeck.com/buch/aposteldekret-und-antikes-vereinswesen-9783161517792?no_cache=1 order@mohrsiebeck.com

Telefon: $+49(0) 7071-923-17$

Telefax: $+49(0) 7071-51104$ 